\title{
Modulation response of quantum dot nanolight-emitting-diodes exploiting purcell- enhanced spontaneous emission
}

Skovgård, Troels Suhr; Gregersen, Niels; Lorke, Michael; Mørk, Jesper

Published in:

Applied Physics Letters

Link to article, DOI:

$10.1063 / 1.3592571$

Publication date:

2011

Document Version

Publisher's PDF, also known as Version of record

Link back to DTU Orbit

Citation (APA):

Skovgård, T. S., Gregersen, N., Lorke, M., \& Mørk, J. (2011). Modulation response of quantum dot nanolightemitting-diodes exploiting purcell-enhanced spontaneous emission. Applied Physics Letters, 98(21), 211109. https://doi.org/10.1063/1.3592571

\section{General rights}

Copyright and moral rights for the publications made accessible in the public portal are retained by the authors and/or other copyright owners and it is a condition of accessing publications that users recognise and abide by the legal requirements associated with these rights.

- Users may download and print one copy of any publication from the public portal for the purpose of private study or research.

- You may not further distribute the material or use it for any profit-making activity or commercial gain

- You may freely distribute the URL identifying the publication in the public portal 


\title{
Modulation response of quantum dot nanolight-emitting-diodes exploiting purcell-enhanced spontaneous emission
}

\author{
T. Suhr, ${ }^{a)}$ N. Gregersen, M. Lorke, and J. Mørk \\ Department of Photonics Engineering, DTU Fotonik, Technical University of Denmark, Ørsteds Plads 343, \\ DK2800 Kgs. Lyngby, Denmark
}

(Received 17 January 2011; accepted 28 April 2011; published online 24 May 2011)

\begin{abstract}
The modulation bandwidth for a quantum dot light-emitting device is calculated using a detailed model for the spontaneous emission including the optical and electronic density-of-states. We show that the Purcell enhancement of the spontaneous emission rate depends critically on the degree of inhomogeneous broadening relative to the cavity linewidth and can improve the modulation speed only within certain parameter regimes. (C) 2011 American Institute of Physics.
\end{abstract}

[doi:10.1063/1.3592571]

The continued increase in data processing speed is limited by the power consumption and heat generation of electrical wiring. It has been suggested instead to exploit optical busses, which in turn require ultrasmall and low-power optical sources that can be modulated at very high speeds. ${ }^{1}$ Nanolight-emitting-diodes (nanoLEDs) are nanostructured light emitting devices and appear as a promising technology that also has the potential of cheap mass fabrication. In these structures, a photonic crystal defect nanocavity is employed to strongly confine the optical mode, thereby enhancing the carrier recombination rate via the Purcell effect. ${ }^{2}$ However, a better understanding of the limitations of these devices is needed. It was recently shown ${ }^{3}$ that in quantum well (QW) nanoLEDs and nanolasers the Purcell enhanced spontaneous emission is severely limited compared to earlier predictions. ${ }^{4,5}$ The limitation originates from the broad-band nature of QW transitions as compared to the narrow optical density of states implied by a high-quality nanocavity. Quantum dots (QDs), on the other hand, have discrete energy states, but are subject to inhomogeneous broadening due to size fluctuations stemming from the fabrication techniques. ${ }^{6}$ In this letter, we report a detailed investigation of the modulation speed of QD based nanoLEDs and establish necessary conditions to achieve high modulation speed. We limit the scope to below threshold operation, where spontaneous emission dominates the recombination processes.

We consider an ensemble of QDs embedded in a high-Q cavity and model the dynamics using the rate equations

$$
\begin{aligned}
& \dot{N}=J-R_{c}-R_{b}, \\
& \dot{S}=\Gamma R_{c}-\frac{S}{\tau_{p}},
\end{aligned}
$$

where $N$ is the total (QD ground level and wetting layer) carrier density, $S$ is the photon density, $J$ is the injection current density, $R_{c}\left(R_{b}\right)$ is the spontaneous emission coupled into the cavity mode (to all other modes), $\Gamma$ is the confinement factor and $\tau_{p}=Q / \omega_{c}$ is the photon life time given by the cavity quality factor and resonance frequency. An important difference from standard rate equations ${ }^{7}$ is that the spontaneous emission is Purcell enhanced and is calculated using a

${ }^{a)}$ Electronic mail: suhr@fotonik.dtu.dk. detailed model of the interaction between a confined optical mode and the possible electronic transitions ${ }^{3,7}$

$$
R_{c}+R_{b}=\iint \rho_{e} f_{2}\left(1-f_{1}\right) A_{21} L \mathrm{dh} \nu \mathrm{dE} .
$$

For the electronic density-of-state (DOS) $\left(\rho_{e}\right)$, a wetting layer and a Gaussian distribution of QD energies with standard deviation $\sigma$ representing inhomogeneous broadening, is taken into account. The factor $A_{21}$ contains the optical DOS $\left(\rho_{o p}\right)$ and is stated in full in Ref. 3. It is also given by $A_{21}$ $=\rho_{o p} h \nu B_{21}$, where $B_{21}$ is the Einstein B coefficient. $\rho_{o p}$ is modeled as a Lorentzian lineshape (times the Purcell enhanced two-state recombination rate in bulk ${ }^{7}$ ) within a photonic band gap and with a bulk background. The homogeneous broadening $(L)$ is modeled as a Lorentzian with fullwidth at half-maximum $\gamma$ and the hole and electron quasiFermi functions $\left(f_{1}\right.$ and $\left.f_{2}\right)$ are determined from the total carrier density. The Purcell enhancement is given by ${ }^{8} F$ $=(6 Q) /\left(\pi^{2} V_{n}\right)$, where $V_{n}$ is the mode volume measured in half wavelengths cubed $[\lambda /(2 n)]^{3}$. An example of the electronic and optical DOS is shown in Fig. 1. Notice that the model assumes local equilibrium between QD and wetting layer states, ${ }^{9}$ which is consistent if the capture time, usually on the order of a few picoseconds or less, ${ }^{10}$ is smaller than the inverse of the modulation frequency predicted by the model. Furthermore, scattering rates need to be low enough,

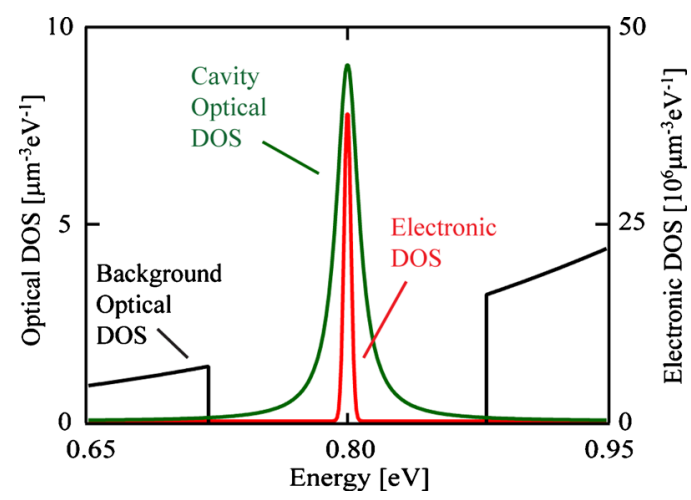

FIG. 1. (Color online) Illustration of the inhomogeneously broadened electronic DOS and the optical DOS. The latter consists of a Lorentzian cavity resonance inside a background DOS with a photonic band gap. The band gap of the wetting layer is $1 \mathrm{eV}$. 


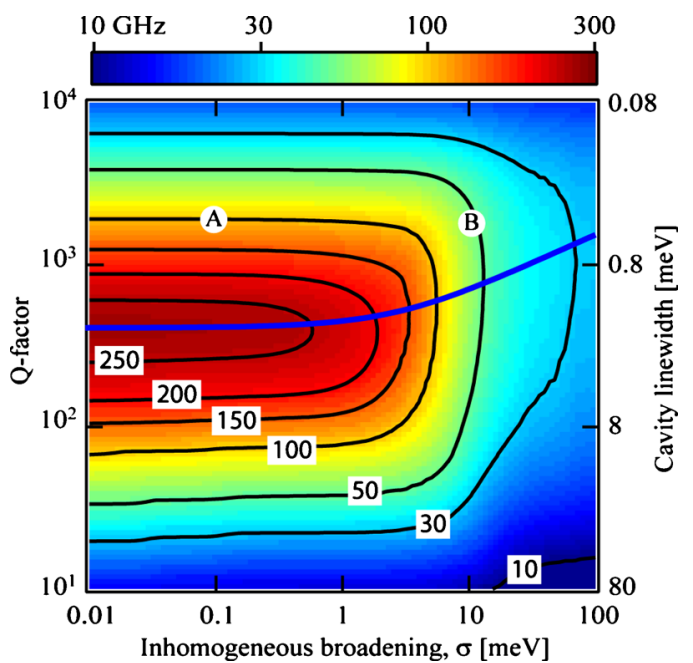

FIG. 2. (Color online) The maximum $3 \mathrm{~dB}$-bandwidth as function of $Q$-factor and inhomogeneous broadening. The contour lines are drawn for constant modulation speed (in gigahertz). The devices A and B, treated in detail in Fig. 3, and the optimum $Q$ are indicated. The parameter values used are listed in Table I.

that the modulation response is not reduced by the scattering itself. $^{11}$

From this model, we calculate the $3 \mathrm{~dB}$-bandwidth below threshold using a small-signal analysis. We set $f_{2}=1$ $-f_{1}=f$ and use the transparency condition $(f=1 / 2)$ as a lower bound for the threshold and plot the maximum modulation speed below this value. The result is displayed in Fig. 2 for a range of $Q$-factors and inhomogeneous widths using the parameters in Table I. We here, choose a value for homogeneous broadening appropriate for low temperature and low density in order to be able to see the effect of inhomogeneous broadening. Two interesting trends are seen: (1) when increasing the $Q$-factor, the $3 \mathrm{~dB}$-frequency goes through a maximum at $Q \sim 500$. The initial rise is due to the Purcell enhancement increase with $Q$. The decrease at large $Q$-factors is an intrinsic feature of all resonators and is due to the photon life time becoming large, prohibiting fast operation. (2) When increasing the inhomogeneous broadening above $\sim 0.5 \mathrm{meV}$, the $3 \mathrm{~dB}$-frequency decreases, whereas it is almost constant below $\sim 0.5 \mathrm{meV}$. Both features can be understood from a small-signal expression for the $3 \mathrm{~dB}$ bandwidth derived from Eqs. (1) and (2)

TABLE I. Parameters used in the calculations. Notice that the differential bulk life time $\left(\tau_{21}\right)$ is the transition time between two states (Ref. 7) and is chosen so the bulk carrier life time is 1 ns.

\begin{tabular}{lc}
\hline \hline \multicolumn{1}{c}{ Parameter } & Value \\
\hline Cavity resonance $\left(\hbar \omega_{0}\right)$ & $0.8 \mathrm{eV}$ \\
Refractive index $(n)$ (see Ref. 7$)$ & 3.4 \\
Mode volume $\left(V_{n}\right)$ (see Ref. 12) & 1 \\
Homogeneous broadening $(\gamma)$ (see Ref. 13) & $100 \mu \mathrm{eV}$ \\
Temperature $(T)$ (see Ref. 13) & $100 \mathrm{~K}$ \\
3D density of dots $\left(\rho_{\mathrm{QD}}\right)($ see Ref. 14) & $2 \times 10^{4} \mu \mathrm{m}^{-3}$ \\
Confinement factor $(\Gamma)$ & 0.01 \\
Photonic band gap $($ see Ref. 15) & $0.2 \mathrm{eV}$ \\
Differential bulk life time $\left(\tau_{21}\right)$ & $125 \mathrm{ps}$ \\
\hline \hline
\end{tabular}

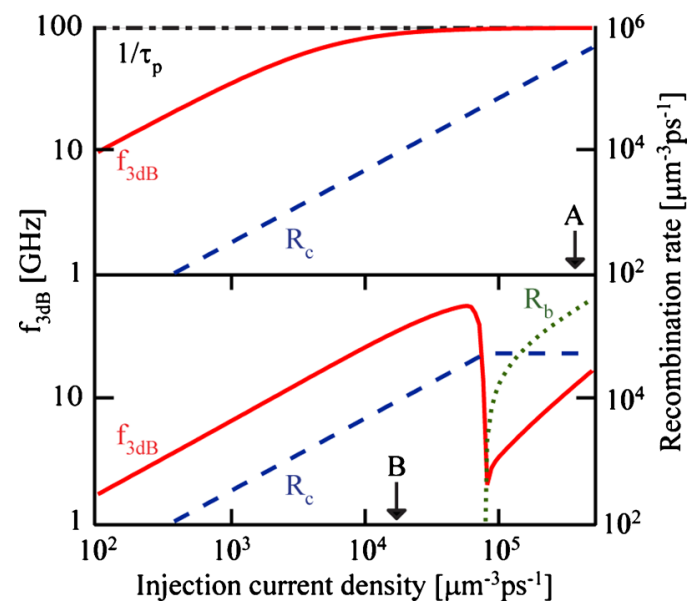

FIG. 3. (Color online) The $3 \mathrm{~dB}$-bandwidth (solid), $R_{c}$ (dashed) and $R_{b}$ (dotted) at $Q=2000$ for an inhomogeneous broadening of $100 \mu \mathrm{eV}$ (device $\mathrm{A}$, top) and $10 \mathrm{meV}$ (device B, bottom). The dot-dashed indicates the photon life time limit and the arrows indicate the transparency current for A and B. For device A, $R_{b}<100 \mu \mathrm{m}^{-3} \mathrm{ps}^{-1}$ and is, therefore, not shown.

$$
f_{3 \mathrm{~dB}}=\frac{1}{2 \pi}\left[\tau_{p}^{2}+\tau_{r}^{2}\right]^{-1 / 2}, \quad \frac{1}{\tau_{r}}=\partial_{N} R_{c}+\partial_{N} R_{b} .
$$

Below transparency, $\tau_{r}$ is well described by $\partial_{N} R_{b}=0$ and $\partial_{N} R_{c}=2 B N_{0}$. Here, $N_{0}$ is the steady state carrier density and $B$ can be approximated by

$$
B=\frac{F}{\tau_{21} \rho_{\mathrm{QD}}} \frac{\Gamma_{p}}{\Gamma_{p}+\gamma+\sigma^{\prime}}, \quad \sigma^{\prime}=\sqrt{2 \ln (2)} \sigma,
$$

where $\tau_{21}$ is the electron-hole recombination time for two states in bulk, ${ }^{7} \rho_{\mathrm{QD}}$ is the three-dimensional (3D) density of dots and $\Gamma_{p}=2 \hbar / \tau_{p}$ is the cavity linewidth. At low $Q$-factors $f_{3 \mathrm{~dB}}$ is governed by $1 / \tau_{r}$, which increases with $Q$, but at high $Q$-factors the bandwidth is limited by the term $\tau_{p}$ $=Q / \omega_{0}$. The $Q$-factor giving the maximum bandwidth is found for $\partial_{Q} f_{3 \mathrm{~dB}}=0$ which for $\gamma+\sigma^{\prime} \ll \Gamma_{p}$ equals (for $N_{0}$ $\left.=\rho_{\mathrm{QD}} / 2\right)$

$$
Q_{\mathrm{opt}}=\sqrt{\frac{\pi^{2}}{6} \omega_{0} \tau_{21} V_{n}} .
$$

In Fig. 2, $Q_{\mathrm{opt}}$ is indicated by the blue line and is seen to deviate from the value in Eq. (6) when $\gamma+\sigma^{\prime}$ becomes comparable to $\Gamma_{p}$. For increasing inhomogeneous broadening, Eqs. (4) and (5) reproduce the decrease observed in Fig. 2 for large $\sigma$.

Figure 3 plots $f_{3 \mathrm{~dB}}$ and the recombination rates against the injection current density $(J)$ for the two devices marked in Fig. 2, one with $\sigma=100 \mu \mathrm{eV}$ (device A) and one with $\sigma$ $=10 \mathrm{meV}$ (device B), both with $Q=2000$. The arrows in Fig. 3 indicate lower bounds on the threshold, namely where $f$ $=1 / 2$, indicating the validity range of the present model. The $3 \mathrm{~dB}$-frequency for device A is seen to increase with $J$ until it becomes limited by the photon life time and the spontaneous recombination is dominated by emission into the cavity. For device $\mathrm{B}, R_{c}$ increases with $J$ until the quasi-Fermi level separation becomes larger than the cavity resonance energy. After this point $R_{c}$ becomes constant and $R_{b}$ rises sharply. This is reflected in $f_{3 \mathrm{~dB}}$ which goes through a maximum and then decreases as $\partial_{N} R_{c} \rightarrow 0$ [see Eq. (4)]. The final increase is due to $R_{b}$ that becomes large when the wetting layer states become important. 


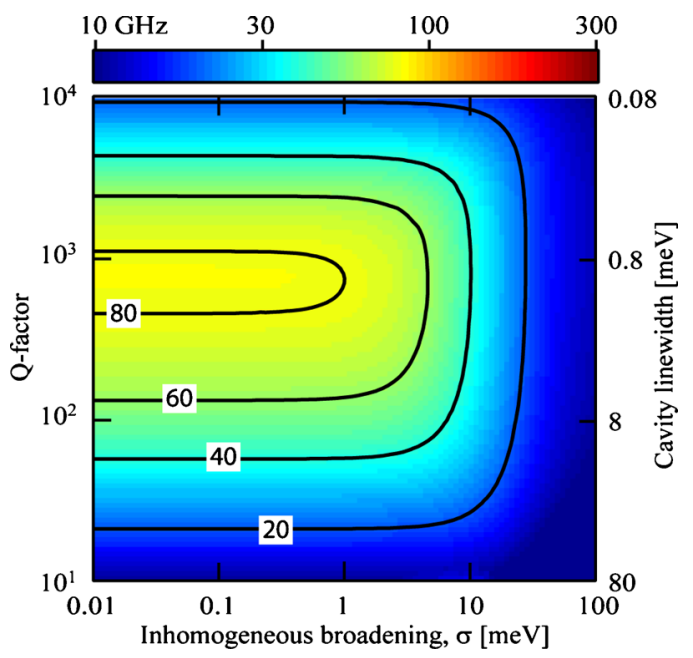

FIG. 4. (Color online) The maximum $3 \mathrm{~dB}$-bandwidth as function of $Q$-factor and inhomogeneous broadening. The contour lines are drawn for constant modulation speed (in gigahertz). The parameters are listed in Table I except that the homogeneous broadening is $10 \mathrm{meV}$ and room temperature is considered.

In Fig. 4 , we plot $f_{3 \mathrm{~dB}}$ for the same range of $Q$-factors and $\sigma$ as in Fig. 2, but for a homogeneous broadening, $\gamma$, of $10 \mathrm{meV}$ corresponding to room temperature operation. ${ }^{13,16}$ The same general dependence on $Q$ and $\sigma$ is seen, but the maximum $3 \mathrm{~dB}$-bandwidth is significantly lower. The modulation bandwidth is seen to become independent of the inhomogeneous broadening, $\sigma$, for $\sigma \ll \gamma$. Note that $f_{3 \mathrm{~dB}}$ continues to increase even for $\sigma<\gamma$ as the width of the distribution is inversely proportional to $\gamma+\sigma$ [see Eq. (5)].

In conclusion, we have calculated the $3 \mathrm{~dB}$-bandwidth for QD nanoLEDs using a detailed model for the spontaneous recombination. It was found that for inhomogeneous broadening smaller than the homogeneous broadening, the modulation bandwidth initially increases with the Purcell en- hancement and is limited by the photon escape rate for high $Q$-factors, giving an optimal $Q$-factor. At large broadenings, the $3 \mathrm{~dB}$ bandwidth is reduced due to the electronic DOS becoming much broader than the optical DOS. Figures 2 and 4 show that with today's technology nanoLEDs are not competitive with conventional devices and improvements of the techniques to produce QD ensembles with low broadening are therefore required in order to unlock the full potential of nanoLEDs.

The authors would like to thank Villum Fonden for financial support via the NATEC (Nanophotonics for TErabit Communications) center.

${ }^{1}$ D. A. B. Miller, Proc. IEEE 97, 1166 (2009).

${ }^{2}$ E. M. Purcell, H. C. Torrey, and R. V. Pound, Phys. Rev. 69, 681 (1946).

${ }^{3}$ T. Suhr, K. Yvind, N. Gregersen, and J. Mørk, Opt. Express 18, 11230 (2010).

${ }^{4}$ H. Altug, D. Englund, and J. Vučković, Nat. Phys. 2, 484 (2006).

${ }^{5}$ E. K. Lau, A. Lakhani, R. S. Tucker, and M. C. Wu, Opt. Express 17, 7790 (2009)

${ }^{6}$ D. Leonard, K. Pond, and P. M. Petroff, Phys. Rev. B 50, 11687 (1994).

${ }^{7}$ L. A. Coldren and S. W. Corzine, Diode Lasers and Photonic Integrated Circuits (Wiley, New York, 1995).

${ }^{8}$ J. Gerard, Single Quantum Dots: Fundamentals, Applications and New Concepts, edited by P. Michler (Springer, New York, 2004), Chap. 7.

${ }^{9}$ T. W. Berg and J. Mørk, IEEE J. Quantum Electron. 40, 1527 (2004).

${ }^{10}$ T. Markussen, P. Kristensen, B. Tromborg, T. W. Berg, and J. Mørk, Phys. Rev. B 74, 195342 (2006).

${ }^{11}$ M. Lorke, T. R. Nielsen, and J. Mørk, Appl. Phys. Lett. 97, 211106 (2010).

${ }^{12}$ M. Nomura, Y. Ota, N. Kumagai, S. Iwamoto, and Y. Arakawa, Appl. Phys. Lett. 97, 191108 (2010).

${ }^{13}$ M. Bayer and A. Forchel, Phys. Rev. B 65, 041308 (2002).

${ }^{14}$ K. Jacobi, Prog. Surf. Sci. 71, 185 (2003).

${ }^{15}$ J. D. Joannopoulos, S. G. Johnson, and J. N. Winn, Photonic Crystals: Molding the Flow of Light (Princeton University Press, New Jersey, 2008).

${ }^{16}$ M. Lorke, T. R. Nielsen, J. Seebeck, P. Gartner, and F. Jahnke, Phys. Rev. B 73, 085324 (2006). 